\title{
Symmetric decomposition of Mueller matrices reveals a new parametric space for polarimetric assistance in colon cancer histopathology
}

Ivanov, Deyan, Dremin, Viktor, Borisova, Ekaterina, Bykov, Alexander, Meglinski, Igor, et al.

Deyan Ivanov, Viktor Dremin, Ekaterina Borisova, Alexander Bykov, Igor Meglinski, Tatiana Novikova, Razvigor Ossikovski, "Symmetric decomposition of Mueller matrices reveals a new parametric space for polarimetric assistance in colon cancer histopathology," Proc. SPIE 11646, Polarized Light and Optical Angular Momentum for Biomedical Diagnostics, 1164614 (5 March 2021); doi: 10.1117/12.2578090

SPIE. Event: SPIE BiOS, 2021, Online Only 


\title{
Symmetric decomposition of Mueller matrices reveals a new parametric space for polarimetric assistance in colon cancer histopathology
}

\author{
Deyan Ivanov $^{\mathrm{a}, \mathrm{b}}$, Viktor Dremin ${ }^{\mathrm{c}, \mathrm{d}}$, Ekaterina Borisova ${ }^{\mathrm{b}}$, Alexander Bykov ${ }^{\mathrm{e}}$, Igor Meglinski $^{\mathrm{c}, \mathrm{e}, \mathrm{f}}$, \\ Tatiana Novikova ${ }^{a}$, and Razvigor Ossikovski ${ }^{a}$ \\ ${ }^{a}$ LPICM, CNRS, Ecole Polytechnique, Institut Polytechnique de Paris, Palaiseau, France \\ ${ }^{b}$ Institute of Electronics, Bulgarian Academy of Sciences, Sofia, Bulgaria \\ ${ }^{c}$ College of Engineering and Physical Sciences, Aston University, Birmingham, UK \\ ${ }^{\mathrm{d}}$ Research \& Development Center of Biomedical Photonics, Orel State University, Russia \\ ${ }^{e}$ Optoelectronics and Measurement Techniques unit, University of Oulu, Finland \\ ${ }^{\mathrm{f}}$ Institute of Clinical Medicine N.V. Sklifosovsky, I.M. Sechenov First Moscow State Medical \\ University, Moscow, Russia
}

\begin{abstract}
Tissue polarimetry could be identified as a complementary optical and non-invasive technique to assist the gold standard histopathology analysis of tissue. In general, polarimetric diagnostics is based on tracing different polarimetric responses (including light depolarization) in tissue zones with structure altered by the benign and pre/cancerous formations. In this manuscript, both healthy and malignant tissue zones of a thick formalin-fixed colon specimen were used for Mueller matrix measurements. Additionally, two more Mueller matrices from Monte Carlo simulation and tissue mimicking phantom were also evaluated, in order to assess polarimetric characterization and modeling of turbid media. Symmetric decomposition algorithm of Mueller matrices developed in house was adopted to extract both polarization and depolarization properties, encoded in the Mueller matrix elements. The decomposition products allowed to reveal important information about the internal tissue structure and morphology. The depolarization and polarization parameters were found to follow the particular trends that depend on a choice of parametric space.
\end{abstract}

Keywords: Tissue polarimetry, Monte Carlo simulation, tissue phantoms, symmetric decomposition, colon cancer

\section{INTRODUCTION}

Within the expanding field of biophotonics ${ }^{1}$ the development of new optical techniques is required that will serve as a support to medical doctors for cancer diagnostics. Such methods should be non-invasive, fast and relatively cheap to implement. Apart from the scalar description of light, characterized by the spatial distribution of light intensity $I(x, y)$ and its phase $\varphi$, optical polarization has vectorial nature and adds another degree of freedom..$^{2}$ The polarized light was found to be sensitive to structural variations of biological tissue. ${ }^{3-7}$ Likewise, tissue polarimetry can provide information about tissue morphology and its spatial heterogeneity, utilizing only polarization of light. Thus, no contrast agents or hazardous ionizing radiation with additional dose load of both patients and staff are needed. Several groups have duly paved their way to implement polarimetric measurements for in vivo or pre/clinical trials. ${ }^{8-11}$ However, prior to translation to clinics a step-by-step approach should be meticulously followed, in order to study in details the diagnostic potential of optical polarization.

Light-tissue interactions are complex phenomena, due to the anisotropic and heterogeneous nature of biological tissues. Hence, polarized Monte Carlo simulations can give better insight to understand polarized light propagation within turbid tissue-like scattering medium. ${ }^{12-15}$ Via modeling it becomes also possible to predict

\footnotetext{
*Correspondence: d.ivanov@ie.bas.bg
} 
light distribution inside the biological specimens ${ }^{16,17}$ and to evaluate malignant, structural variations in tissues. ${ }^{18}$ On the other hand, as the polarization of light in turbid media is randomized quickly due to multiple scattering events, Monte Carlo modeling can be used for the calculations of Mueller matrix elements and Stokes vector of partially depolarized light.$^{19,20}$ An auxiliary approach for validating simulations and for mimicking desired optical properties of biological specimens, would include fabrication of tissue phantoms that can be used accordingly in polarimetry. ${ }^{21-24}$

Straightforward interpretation of tissue polarimetric properties directly from Mueller matrix elements would be applicable to homogeneous and isotropic samples only. ${ }^{25}$ For anisotropic samples a complementary Mueller matrix decomposition techniques are required, ${ }^{26-28}$ without violation of the condition of the physical realizability of Mueller matrix. ${ }^{29}$ In this work we present the results of comparative studies of various turbid media, in terms of its depolarization capabilities. Four examples of highly scattering media were selected for Mueller polarimetry studies, involving a virtual phantom (Mueller matrix was calculated using polarized Monte Carlo algorithm), real phantom and healthy and malignant zones of ex vivo thick formalin-fixed colon specimen. The Mueller matrices of real samples were measured experimentally. Extracted via symmetric decomposition of Mueller matrix, their intrinsic depolarization properties were analyzed, compared, and related to the scattering properties and inner structure of each sample.

\section{THEORY}

The description of light with arbitrary degree of polarization $\rho$ has been abundantly described elsewhere. ${ }^{30}$ Here, in terms of brevity an alternative definite convention is introduced: ${ }^{31}$

$$
\mathbf{S}=I(1, \mathbf{p})^{T}, \quad \mathbf{p}=\rho \mathbf{u}, \quad \mathbf{u}=[\cos (2 \theta) \cos (2 \epsilon), \sin (2 \theta) \cos (2 \epsilon), \sin (2 \epsilon)]^{T},
$$

where in Eq. $1 \mathbf{p}$ and $\mathbf{u}$ are the polarization and Poincaré vectors respectively, the parameters $\rho \in[0 \leq \rho \leq 1]$, $\theta \in[-\pi / 2 \leq \theta \leq \pi / 2]$ and $\epsilon \in[-\pi / 4 \leq \epsilon \leq \pi / 4]$. For any non-over polarizing media (i.e. $\rho \leq 1)$ there is a corresponding $4 \times 4$, real transfer function that transforms linearly $\mathbf{S}_{\text {in }}$ into $\mathbf{S}_{\text {out }}$. In order to measure a complete Mueller matrix, at least four input and four output polarization co-variations are required. Eventually, one needs to solve a system of four linear equations to derive the columns of $\mathbf{M} i j$ for each $i:{ }^{32}$

$$
\mid \begin{gathered}
S_{H / V}^{o u t}=M_{i 1} \pm M_{i 1 / 2} \\
S_{P / R}^{o u t}=M_{i 1}+M_{i 3 / 4}
\end{gathered}
$$

where $\mathrm{H} / \mathrm{V}$ denote horizontal/vertical, $\mathrm{P} / \mathrm{R}$ denote $+45^{\circ} /$ right circular polarization states, and $i, j=\in[1: 4]$. Subject to data noise and/or experimental errors, each matrix $\mathbf{M} i j$ needs to be filtered to obey the criteria of physical realizability. Then symmetric decomposition of Mueller matrix can be applied to linear or arbitrary elliptical retardance and/or diattenuation as well and it is well suited for angular-resolved measurements: ${ }^{26-28}$

$$
\mathbf{M}=\mathbf{M}_{\mathbf{D}_{2}} \mathbf{M}_{\mathbf{R}_{2}} \mathbf{M}_{\Delta} \mathbf{M}_{\mathbf{R}_{1}} \mathbf{M}_{\mathbf{D}_{1}}
$$

where $\mathbf{M}_{\Delta}, \mathbf{M}_{\mathbf{R}}$ and $\mathbf{M}_{\mathbf{D}}$ denote the Mueller matrices of a depolarizer $\Delta$ with the depolarization coefficients $\mathrm{d}_{\mathrm{i}}$, a retarder $\mathbf{R}$ with phase shift $\varphi$ and a diattenuator $\mathbf{D}$ with diattenuation $\mathbf{D}$. Their explicit forms are defined as: ${ }^{30}$

$$
\mathbf{M}_{\Delta}=\operatorname{diag}\left(1, d_{1}, d_{2}, d_{3}\right), \quad \mathbf{M}_{\mathbf{R}}=\left[\begin{array}{cc}
1 & \overrightarrow{0}^{T} \\
\overrightarrow{0} & m_{\varphi}
\end{array}\right], \quad \mathbf{M}_{\mathbf{D}}=\left[\begin{array}{cc}
1 & \vec{D}^{T} \\
\vec{D} & m_{D}
\end{array}\right]
$$

In the above equations, for shortening both sub-matrices $\mathbf{m}_{\varphi}$ and $\mathbf{m}_{\mathbf{D}}=\operatorname{diag}\left(1, \sqrt{1-D^{2}}, \sqrt{1-D^{2}}\right)$ were introduced and are valid for linear polarization properties. From Eq.(4) it becomes straightforward to find D, whereas $\Delta \in[0 \leq \Delta \leq 1]$ and $\varphi \in[0 \leq \varphi \leq \pi]$ can be calculated $\operatorname{as}^{30}$ :

$$
\Delta=1-\frac{\sum_{i=1}^{3}\left|d_{\mathrm{i}}\right|}{3}, \quad \varphi=\cos ^{-1}\left[\frac{\operatorname{tr}\left(\mathbf{m}_{\varphi}\right)-1}{2}\right] .
$$




\section{MONTE CARLO MODEL}

At every moment $t$ and at each point $\mathbf{r}$ inside a scattering medium the intensity $d I$ of the light propagating in the solid angle $d \Omega$ around the direction defined by the unit vector $\vec{\Omega}$ is given by: ${ }^{33}$

$$
d I(\mathbf{r}, \vec{\Omega}, t)=L(\mathbf{r}, \vec{\Omega}, t) d \Omega
$$

where $L$ is the radiance, which evolution can be expressed as follows:

$$
\frac{1}{v} \frac{\partial L(\mathbf{r}, \vec{\Omega}, t)}{\partial t}+\vec{\Omega} \nabla L(\mathbf{r}, \vec{\Omega}, t)=\mu_{\mathrm{t}} L(\mathbf{r}, \vec{\Omega}, t)+\int_{\Omega^{\prime}} \mu_{\mathrm{s}} p\left(\overrightarrow{\Omega^{\prime}} \rightarrow \vec{\Omega}\right) L\left(\mathbf{r}, \overrightarrow{\Omega^{\prime}}, t\right) d \Omega^{\prime}+\sum(\mathbf{r}, \vec{\Omega}, t)
$$

where $v=c / n_{m}$ is the velocity of light in a medium with the refractive index $n_{m}, \mu_{\mathrm{t}}=\mu_{\mathrm{a}}+\mu_{\mathrm{s}}$ is the total extinction coefficient, $\mu_{\mathrm{a}}$ and $\mu_{\mathrm{s}}$ are the absorption and scattering coefficients respectively, $p\left(\overrightarrow{\Omega^{\prime}} \rightarrow \vec{\Omega}\right)$ is the phase function and $\sum(r, \vec{\Omega}, t)$ is the radiance of the internal sources (if any). The scalar intensity $I$ can be replaced by defining a vectorial radiance $\mathbf{L}$ with the components of Stokes vector:

$$
d S_{i}(\mathbf{r}, \vec{\Omega}, t)=d L_{i}(\mathbf{r}, \vec{\Omega}, t) d \Omega, \quad i=[0,3]
$$

Hence, the vectorial radiance $\mathbf{L}$ is the probability density function of the Stokes vector components in $\Omega$ space. Thus, the new phase function $\mathbf{P}$, is an angular dependent $4 \times 4$ real matrix that depends on the input vectorbased radiance $\mathbf{L}$, due to normalization conditions. The propagation of polarized light in scattering media can be described by the integro-differential vector radiative transfer equation (VRTE) that represents the energy conservation law for the Stokes vector:

$$
\frac{1}{v} \frac{\partial \mathbf{L}(\mathbf{r}, \vec{\Omega}, t)}{\partial t}+\vec{\Omega} \nabla \mathbf{L}(\mathbf{r}, \vec{\Omega}, t)=\mu_{\mathrm{t}} \mathbf{L}(\mathbf{r}, \vec{\Omega}, t)+\int_{\Omega^{\prime}} \mu_{\mathrm{s}} \mathbf{P}\left(\overrightarrow{\Omega^{\prime}} \rightarrow \vec{\Omega}\right) \mathbf{L}\left(\mathbf{r}, \overrightarrow{\Omega^{\prime}}, t\right) d \Omega^{\prime}+\sum(\mathbf{r}, \vec{\Omega}, t)
$$

The integral form of the transport equation can be expressed as:

$$
\begin{gathered}
\mathbf{L}(\mathbf{r}, \vec{\Omega}, t)=\int_{0}^{\infty} \exp [-\zeta] d \zeta \\
\zeta=\int_{0}^{s} \mu_{\mathrm{t}}\left(\mathbf{r}-s^{\prime \prime} \vec{\Omega}\right) d s^{\prime \prime}\left[\mathbf{q}\left(\mathbf{r}_{0}-s^{\prime} \vec{\Omega}, \vec{\Omega}, t_{0}-\frac{s^{\prime}}{v}\right)\right] \\
q(\mathbf{r}, \vec{\Omega}, t)=\int_{\Omega^{\prime}} \mu_{\mathrm{S}} \mathbf{P}\left(\overrightarrow{\Omega^{\prime}} \rightarrow \vec{\Omega}\right) \mathbf{L}\left(\mathbf{r}, \overrightarrow{\Omega^{\prime}}, t\right) d \Omega^{\prime}+\sum(r, \vec{\Omega}, t)
\end{gathered}
$$

In Eq. 10c $\mathrm{q}(\mathbf{r}, \vec{\Omega}, \mathrm{t})$ is the total rate at which polarized photons appear at point $(\mathbf{r}, \vec{\Omega}, \mathrm{t})$, because of collisions, as well as of internal and external sources. In the absence of internal sources the integral of Eq. 10c can be estimated by the Monte Carlo technique. The simulation was performed for a sequence of $10^{6}$ photons. The photon's random walk within the scattering medium came to an end either when the photon had hit the detector or had left the computational domain. A 1-cm-thick layer of scattering medium was modelled with spherical scatterers of radius $0.2 \mu \mathrm{m}$, absorption coefficient $\mu_{\mathrm{a}}=0$, scattering coefficient $\mu_{\mathrm{s}}=10 \mathrm{~cm}^{-1}$, refractive index of the scatterers $n_{\mathrm{p}}=1.59$, refractive index of the host medium $n_{\mathrm{h}}=1.33$, wavelength of the incident light $\lambda=633 \mathrm{~nm}$. The following geometrical considerations were chosen, in order to represent the real experimental 
set-up of the measurements: flat-surface sample, illuminated at an oblique angle of $55^{\circ}$ and a detector with the diameter of $1 \mathrm{~mm}$, positioned at $30^{\circ}$ with respect to the normal to the surface of scattering medium. The polarized photons are emitted by an external light source, whereas their propagation is contemplated ballistically between two scattering events over a distance that is calculated from the value of scattering coefficient $\mu_{\mathrm{s}}$. Each elastic scattering event changes both the polarization and the direction of propagation of a scattered photon. New polarization state and scattering angle are calculated by applying the Mie solution for light scattering on a sphere. At the interfaces both reflection and refraction were calculated according to the Fresnel laws. Fixing the input Stokes vector allows us to calculate the corresponding output Stokes vector by averaging over the simulated output polarization states of photons that had reached the detector. This procedure was performed until a variance threshold of $1.5 \%$ was reached for the output Stokes vector components. By repeating the procedure for four different input Stokes vectors $\left(\mathbf{S}_{\mathbf{H}}, \mathbf{S}_{\mathbf{V}}, \mathbf{S}_{\mathbf{P}}\right.$ and $\left.\mathbf{S}_{\mathbf{R C P}}\right)$, the Mueller matrix $\mathbf{M}_{\mathbf{M C}}$ of the virtual phantom was obtained from a resulting system of 16 linear equations.

\section{SAMPLE CHARACTERISTICS}

\subsection{Physical phantom}

The phantom used in this study represents a layer of $2 \mathrm{~mm}$ thickness with scattering coefficient $\mu_{\mathrm{s}}=10 \mathrm{~mm}^{-1}$, absorption coefficient $\mu_{\mathrm{a}}=0.05 \mathrm{~mm}^{-1}$ and anisotropy factor $g=0.8$. The detailed description of the manufacturing and characterization of biological tissue phantoms is given in. ${ }^{34-37}$ A polyvinyl chloride (PVC)-based matrix was used as a transparent host for $Z n O$ nanoparticles that scatter incident light. The required volume fraction of added nanoparticles was estimated on a basis of Mie theory taking into account the polydisperse distribution of particle sizes. The average diameter of the particles was $\sim 0.34 \mu \mathrm{m}$. To control the absorption coefficient of fabricated phantom, a black plastic color composed of CI Pigment Black 7 was added. The optical properties of fabricated phantom including absorption and scattering coefficients as well as the anisotropy factor in the visible and near-infrared spectral range were derived on the basis of the inverse adding-doubling method from the diffuse reflectance, diffuse transmittance and collimated transmittance measurements performed with the spectrophotometer (Gooch \& Housego, USA) equipped with the integrating spheres. ${ }^{36,38}$ Additionally, the refractive index of the phantom was measured using the multiwavelength Abbe refractometer (Atago, Japan). In prior studies it was shown, that this type of phantoms can be considered as a relevant model of biological tissue for red and infrared spectral range.

\subsection{Ex vivo colon specimen}

Thick formalin-fixed ex vivo colon specimen was used for polarimetric experiements. The biological specimen was provided after excision and diagnosis of the pathologist's (G2-Adenocarcinoma) from the University Hospital Tzaritza Yoanna-ISUL(Sofia, Bulgaria). The approval of the hospital ethical committee was obtained for these studies. Two non-adjacent tissue zones (healthy and tumorous) were probed with polarized light, taking as a reference the results of tissue histology analysis. The thickness of the specimen was several millimeters and both zones were characterized by high opacity. Consequently, the polarimetric measurements were done in reflection configuration. Each zone of interest of the specimen was measured 10 times in a sequence and then averaged, in order to improve the reproducibility of the measurement results.

\section{EXPERIMENTAL STUDIES}

The schematic of the experimental set-up is shown in Figure 1. In accordance with the diagnostic window $(600-1300 \mathrm{~nm})$, a probing wavelength of $635 \mathrm{~nm}$ was selected. The conventional polarimetry measurements require a low coherent light source, due to the necessity to avoid the formation of laser speckle. For this particular reason, a supercontinuum fiber laser with low spatial, but high temporal coherence was coupled with an acousto-optical tunable filter (AOTF). All input polarization states were generated with a single half-wave plate or in a combination with a liquid crystal quarter-wave plate, modulated by the applied voltage. Light backscattered from the samples was collected and passed through a spatial filter for a more precise separation of the signal from different tissue zones. Additionally, in-focus measurements were performed with the help of inserted matrix photodetector. All measurements were performed at the angles of incidence and detection $55^{\circ}$ and $30^{\circ}$, respectively, alongside the adopted technique of rotating quarter wave-plate and fixed linear polarizer, contained 
in a commercially available polarimetric device (Thorlabs, USA, PAX5710VIS-T). All Stokes parameters were derived from the Fourier transformation of the detected signal from a photodiode.

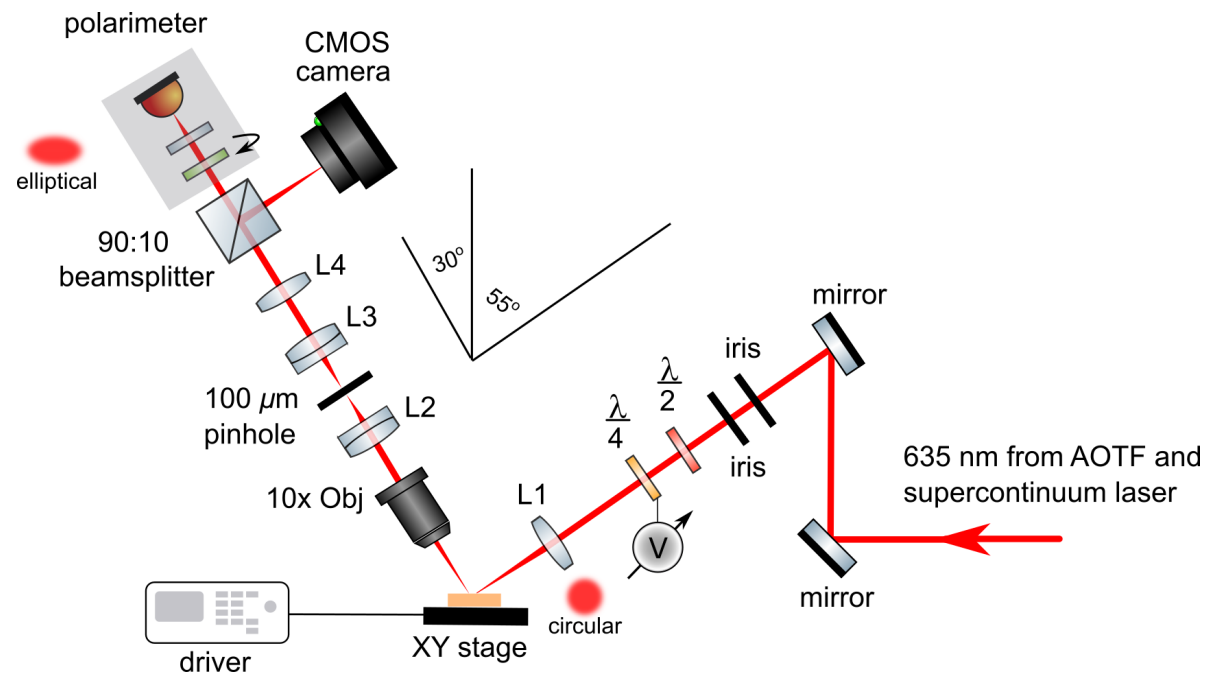

Figure 1: Schematic representation of the experimental set-up.

\section{RESULTS AND DISCUSSION}
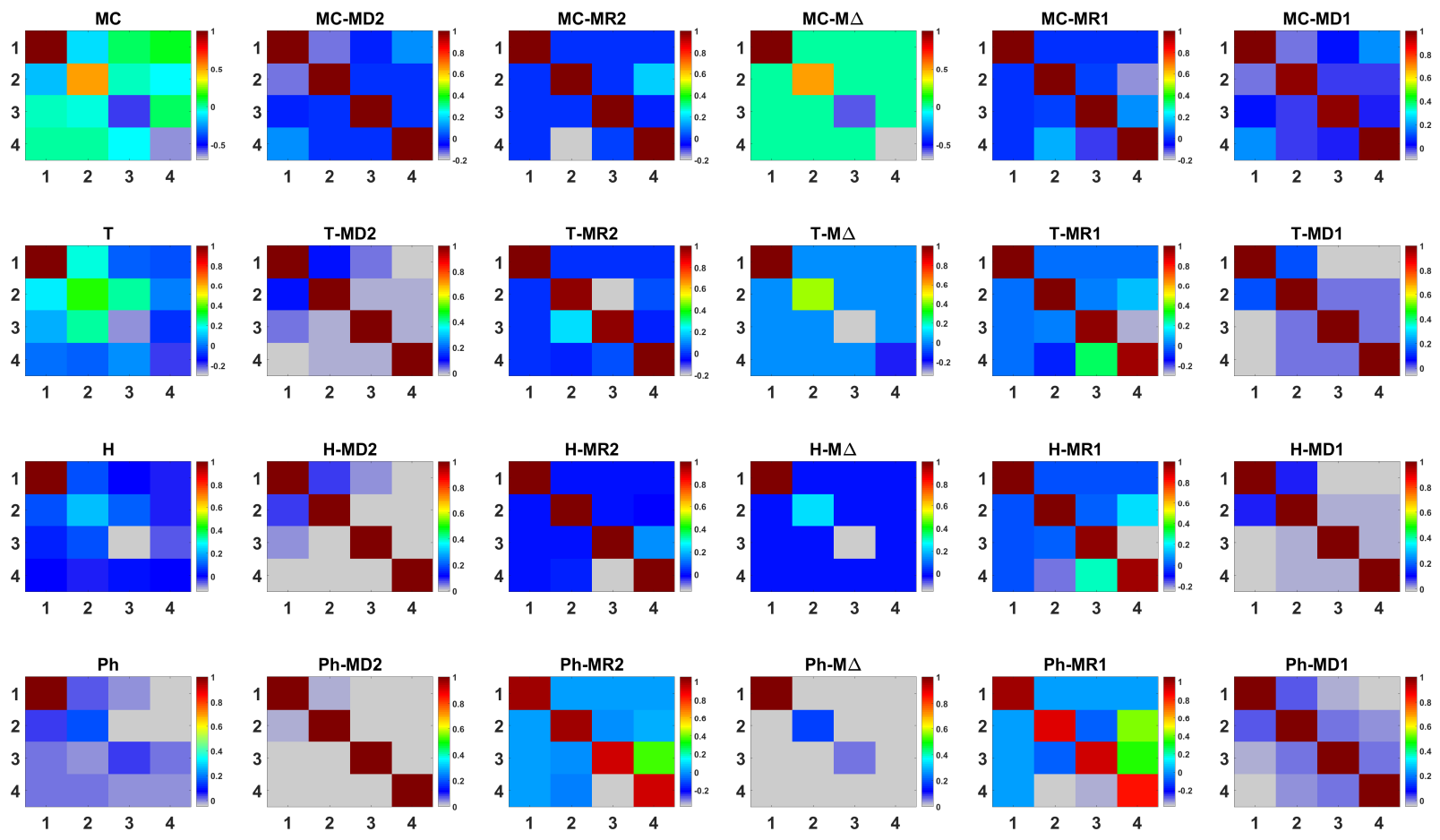

Figure 2: Simulated and measured Mueller matrices (left column) and their symmetric decompositions: Monte Carlo (MC, first row), Tumor colon ( $\mathrm{T}$, second row), Healthy colon (H, third row) and Phantom (Ph, forth row).

We start with the comparison between the polarimetric properties of the simulated phantom and that of the real samples. The inner structure of virtual phantom is comprised of uniformly distributed spherical scatterers of 
fixed size and refractive index within optically isotropic host medium. On the contrary, for all physical samples used in this study the above mentioned assumptions do not hold. Colon specimen tissue contains scattering particles of various size, most of them are non-spherical and have fluctuating refraction index. Thus comparing the simulated Mueller matrix of virtual phantom with the measured Mueller matrices of real samples, it becomes possible to compare the optical properties of the corresponding scattering media.

First, the general form of all Mueller matrices is very close to that of a diagonal depolarizer. This is especially noticeable for the simulated Mueller matrix of virtual phantom. However, the off-diagonal elements are nonzero, which in turn is an indication of other polarimetric properties encoded in the Mueller matrices. Second, a rotational symmetry of reflection would yield an equality $\mathrm{M}_{22}=-\mathrm{M}_{33}$, whereas in Fig. 2 one can easily observe a violation of this criterion for the Mueller matrix of real phantom. Two potential reasons for absence of rotational symmetry could be identified as presence of non-spherical particles, which are not isotropically oriented. Both polarization and depolarization properties of all turbid media can be analyzed after applying the symmetric decomposition algorithm to their corresponding Mueller matrices. The results of symmetric decomposition of simulated and measured matrices are presented in Fig. 3. We denote by $\mathbf{I}$ the identity matrix, such as $\mathbf{M} i \boldsymbol{j}=\delta i j$ and by ID the Mueller matrix of an ideal depolarizer. The former has only polarization and no depolarization properties, while the latter has only depolarization properties.

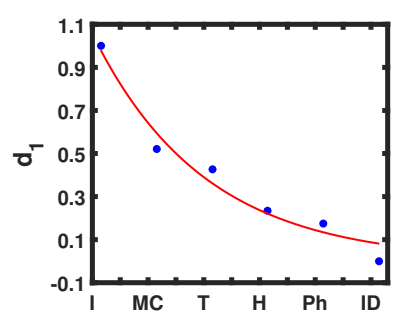

(a)

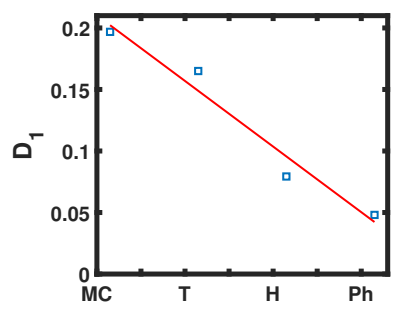

(e)

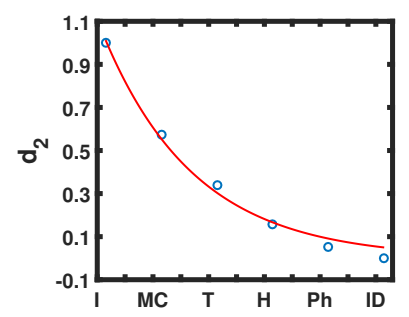

(b)

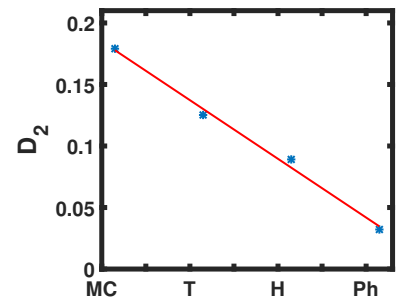

(f)

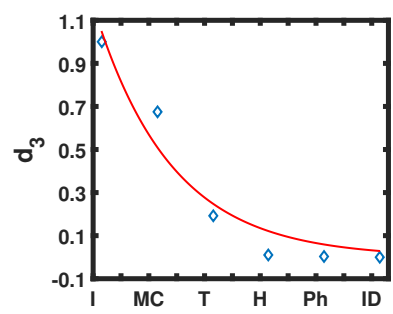

(c)

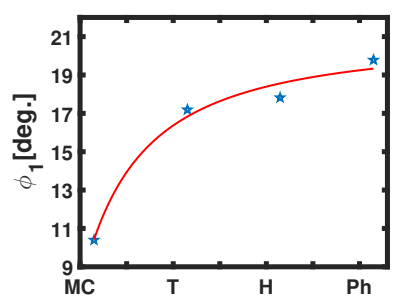

$(\mathrm{g})$

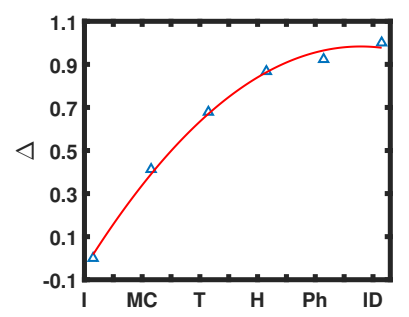

(d)

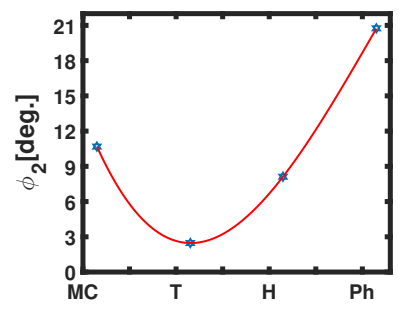

(h)

Figure 3: Symmetric decomposition products: a), b), c) depolarization indices $-\mathrm{d}_{\mathrm{i}}$; d) net depolarization index $-\Delta ; \mathrm{e}), \mathrm{f})$ diattenuadtion $\mathrm{D}_{\mathrm{i}}$ and $\left.\left.\mathrm{g}\right), \mathrm{h}\right)$ retardance $-\varphi_{\mathrm{i}}\left(\phi_{\mathrm{i}}\right.$ and $\varphi_{\mathrm{i}}$ used interchangeably).

In Fig. 3 all red lines serve as a guide-to-the-eye and should not be interpreted as the fit curves, since only categorical variables are presented on the $x$-axes. Thus, it becomes possible to assume a monotonic decrease of all depolarization coefficients $d_{i}$ and a monotonic increase for the depolarization index $-\Delta$. As expected, the simulation predicts less depolarization compared to real samples. Most important, the healthy tissue excels the depolarization of light in comparison with the malignant tissue due to different amount of scattering events in healthy and tumor zones. As for $D_{i}$, clearly there is a linear trend presented, while $R_{1}$ and $R_{2}$ are characterized by monotonic increase and non-linear tendency respectively. Nevertheless, several important tissue properties can be elicited. Namely, there is an increased diattenuation value for the tumor tissue and slightly higher retardance value for the healthy one. In Table 1 all numerical values of the decomposition quantities are summarized.

To sum up, the polarimetric properties of virtual phantom are closer to that of colon tumor tissue, while the polarimetric properties of real phantom are closer to the properties of healthy colon tissue. Control of this phenomenon was achieved by simulating isotropic host medium with ideally spherical scatterers. Also, by setting 


\begin{tabular}{|l|l|l|l|l|l|l|l|l|}
\hline & $\mathbf{d}_{\mathbf{1}}$ & $\mathbf{d}_{\mathbf{2}}$ & $\mathbf{d}_{\mathbf{3}}$ & $\Delta$ & $\mathbf{D}_{\mathbf{1}}$ & $\mathbf{D}_{\mathbf{2}}$ & $\varphi_{\mathbf{1}}$ [deg.] & $\varphi_{\mathbf{2}}$ [deg.] \\
\hline $\mathbf{M C}$ & 0.52 & 0.57 & 0.69 & 0.41 & 0.19 & 0.18 & 10.39 & 10.69 \\
\hline $\mathbf{T}$ & 0.43 & 0.34 & 0.19 & 0.68 & 0.17 & 0.13 & 17.17 & 2.47 \\
\hline $\mathbf{H}$ & 0.24 & 0.16 & 0.01 & 0.87 & 0.08 & 0.09 & 17.81 & 8.14 \\
\hline $\mathbf{P H}$ & 0.17 & 0.05 & 0.004 & 0.93 & 0.05 & 0.03 & 19.77 & 20.73 \\
\hline
\end{tabular}

Table 1: Supplementary table associated with decomposition data ( $\phi_{\mathrm{i}}$ and $\varphi_{\mathrm{i}}$ used interchangeably).

the scattering coefficient of the real phantom ten times higher in comparison to the virtual one would result in an decreased photon mean free path between two scattering events. Consequently, growth in the scattering events would lead to an increased randomization of the initial polaraization state and higher loss of polarization degree. Thus, all real samples are characterized by significant depolarization, with inequality relation of $\mathrm{d}_{1}>\mathrm{d}_{2}>$ $\mathrm{d}_{3}$. Such outcome is an indicator of higher depolarization capabilities for circularly polarized light compared to linear polarized light - typical signature of the Rayleigh scattering regime. On the price of less data processing, the Lu-Chipman decomposition could be applied, but in that case the depolarizer would be "contaminated" with polarizance. To avoid this obstacle, the symmetric decomposition provides an opportunity to extract pure canonical depolarizer and two additional parameters, which define a new parametric space, characterized by diattenuation and retardance. For angular-resolved measurements, the latter two non-redundant parameters give rise to impure diagonal form of all Mueller matrices. Increased diattenuation of tumor tissue would indicate an alteration of the two orthogonal electric field amplitudes $\mathbf{E}_{x / y}$ of partially polarized light without introducing a phase shift between them, and, thus, introducing repolarizing effect. On the other hand, healthy colon tissue possesses slightly higher retardance compared to tumor colon tissue. It could be associated with a disruption of collagen fibrous structure of the extra-cellular matrix, due to the growth of tumor. Finally, a substantial difference in $\Delta_{\varphi}=\left|\varphi_{1}-\varphi_{2}\right|$ is observed for the colon sample. Such an occurrence arises from various reasons as: backscattering configuration with different angles of incidence and detection; difference in the optical path-lengths of both probing and emerging light beams; samples' anisotropy and fibrous structure.

\section{CONCLUSION}

Nowadays, there is a need to improve the efficacy of cancer theranostics, as such necessity would significantly increase life expectancy. For this particular reason, biophotonics can provide adequate assistance to medical doctors and complete the conventional theranostics in medicine. The purpose of current studies is focused mainly on a diagnostic assistance to pathologists on avail of a vectorial light property. Characterizing turbid media only with polirized light has a potential as a complementary approach in histopathology, but is nonetheless far from being a trivial task to implement. Therefore, supplementary techniques such as polarized Monte Carlo modeling and phantom preparation and characterization could enrich and reinforce the experimental studies. Likewise in the current paper, polarized Monte Carlo simulations were adopted to mimic closely the colon tumor tissue, while the phantom provided better proximity to the healthy colon tissue zone. All experimental Mueller matrices were obtained and filtered for data noise and/or experimental errors, whereas their polarimetric properties were extracted with the aid of symmetric decomposition algorithm. The results indicate significant light depolarization with higher contribution to circularly polarized light. Non-negligible diattenuaton and retardance values were also detected both of which were obtained after the application of the symmetric decomposition as well. $\mathrm{D}_{\mathrm{i}}$ and $\varphi_{\mathrm{i}}$ additionally contribute to changes of the probing and emerging light beams polarization states and can be used as diagnostic markers in case of a backscattered configuration. Whenever the diattenuation is insignificant, only intrinsic depolarization is sufficient to be considered. On the contrary, as in our case for the simulated phantom and the tumor tissue, a substantial diattenuation is at presence. Thus, there is a revelation to extrinsic depolarization case. ${ }^{39,40}$ However, such a distinction is beyond the scope of the current manuscript. Hence, a future study focused entirely on the depolarization metrics would be reasonably justified and could enrich the tissue polarimetry appraoches for complementary diagnostic analysis. 


\section{ACKNOWLEDGMENTS}

All authors acknowledge the contribution to Dr. Pengcheng Li to Monte Carlo modeling. Deyan Ivanov acknowledges the PhD fellowship funding by the Doctoral School of Institut Polytechnique de Paris. Dr. Victor Dremin kindly acknowledges personal support from the Russian Science Foundation (projects No. 19-79-00082, experimental data collection). This work received funding from NSF of Bulgaria under project \#KP06-N28/11/14.12.18 "Novel bio-optical methods for determination of chemical and conformational reorganization of collagen-based tissue structures in vitro and in vivo"; The ATTRACT project funded by the EC under Grant Agreement 777222; Academy of Finland (grants 314639 and 325097) and INFOTECH strategic funding.

\section{REFERENCES}

[1] Meglinski, I., [Biophotonics for Medical Applications], Woodhead Publishing, Cambridge (2015).

[2] Bliokh, K. Y., Rodriguez-Fortuno, F. J., Nori, F., and Zayats, A. V., "Spin-orbit interactions of light," Nat. Photonics 9, 796:808 (2015).

[3] Ghosh, N. and Vitkin, I., "Tissue polarimetry: concepts, challenges, applications, and outlook," J. Biomed. Opt. 16, 110801 (2011).

[4] Ivanov, D., Dremin, V., Bykov, A., Borisova, E., Genova, T., Popov, A., Novikova, T., Ossikovski, R., and Meglinski, I., "Colon cancer detection by using Poincaré sphere and 2D polarimetric mapping of ex vivo colon samples," J. Biophotonics 13(8), e202000082 (2020).

[5] Borovkova, M., Bykov, A., Popov, A., Pierangelo, A., Novikova, T., Pahnke, J., and Meglinski, I., "Evaluating $\beta$-amyloidosis progression in Alzheimer's disease with Mueller polarimetry," Biomed. Opt. Express 11(8), 4509-4519 (2020).

[6] Nishizawa, N., Al-Qadi, B., and Kuchimaru, T., "Angular optimization for cancer identification with circularly polarized light," J. Biophotonics, e202000380 (2020).

[7] Novikova, T., Meglinski, I., Ramella-Roman, J. C., and Tuchin, V. V., "Polarized light for biomedical applications," J. Biomed. Opt. 21(7), 071001 (2016).

[8] Kupinski, M., Boffety, M., Goudail, F., Ossikovski, R., Pierangelo, A., Rehbinder, J., Vizet, J., and Novikova, T., "Polarimetric measurement utility for pre-cancer detection from uterine cervix specimens," Biomed. Opt. Express 9(11), 5691-5702 (2018).

[9] Ghassemi, P., Lemaillet, P., Germer, T., Shupp, J., Venna, S., Boisvert, M., Flanagan, K., Jordan, M., and Ramella-Roman, J. C., "Out-of-plane Stokes imaging polarimeter for early skin cancer diagnosis," $J$. Biomed. Opt. 17(7), 076014 (2012).

[10] Feehan, J., Tripodi, N., Fraser, S., Mikkelsen, K., Thewlis, A., Kiatos, D., Husaric, M., and Apostolopoulos, V., "Polarized light therapy: Shining a light on the mechanism underlying its immunomodulatory effects," J. Biophotonics 13(3), e201960177 (2019).

[11] Dremin, V., Marcinkevics, Z., Zherebtsov, E., Popov, A., Grabovskis, A., Kronberga, H., Geldnere, K., Doronin, A., Meglinski, I., and Bykov, A., "Skin complications of diabetes mellitus revealed by polarized hyperspectral imaging and machine learning," IEEE Trans. Med. Imaging (2021).

[12] Kuzmin, V. and Meglinski, I., "Numerical simulation of coherent back-scattering and temporal intensity correlations in random media," Quantum. Electron. 36, 990-1002 (2006).

[13] Kuzmin, V. and Meglinski, I., "Coherent effects of multiple scattering for scalar and electromagnetic fields: Monte-Carlo simulation and milne-like solutions," Opt. Commun. 273, 307-310 (2006).

[14] Doronin, A., Macdonald, M., and Meglinski, I., "Propagation of coherent polarized light in highly scattering turbid media," J. Biomed. Opt. 19, 60502 (2014).

[15] Doronin, A., Radosevich, A., Backman, V., and Meglinski, I., "Two electric field Monte Carlo models of coherent backscattering of polarized light," J. Opt. Soc. Am. A 31, 2394-2400 (2014).

[16] Dremin, V., Zherebtsov, E., Bykov, A., Popov, A., Doronin, A., and Meglinski, I., "Influence of blood pulsation on diagnostic volume in pulse oximetry and photoplethysmography measurements," Appl. Opt. 58, 9398-9405 (2020).

[17] Doronin, A. and Meglinski, I., "Online object oriented Monte Carlo computational tool for the needs of biomedical optics," Biomed. Opt. Express 2, 2461-2469 (2011). 
[18] Kunnen, B., Macdonald, C., Doronin, A., Jacques, S., Eccles, M., and Meglinski, I., "Application of circularly polarized light for non-invasive diagnosis of cancerous tissues and turbid tissue-like scattering media," J. Biophotonics 8, 317-323 (2015).

[19] Gangnus, S., Matcher, S. J., and Meglinski, I., "Monte Carlo modeling of polarized light propagation in biological tissues," Laser Phys. 14, 886-891 (2004).

[20] Antonelli, M. R., Pierangelo, A., Novikova, T., Validire, P., Benali, A., Gayet, B., and De Martino, A., "Mueller matrix imaging of human colon tissue for cancer diagnostics: how Monte Carlo modeling can help in the interpretation of experimental data," J. Biomed. Opt. 18, 10200-10208 (2010).

[21] Agarwal, N., Yoon, J., Garcia-Caurel, E., Novikova, T., Vanel, J.-C., Pierangelo, A., Bykov, A. Popov, A., Meglinski, I., and Ossikovski, R., "Spatial evolution of depolarization in homogeneous turbid media within the differential Mueller matrix formalism," Opt. Lett. 40, 5634-5637 (2015).

[22] Popov, A., Sieryi, O., Kalchenko, V., Bykov, A., and Meglinski, I., "Tissue-mimicking phantoms for biomedical applications," Proc. SPIE 11363, 1136312 (2020).

[23] Dremin, V., Anin, D., Sieryi, O., Borovkova, M., Näpänkangas, J., Meglinski, I., and Bykov, A., "Imaging of early stage breast cancer with circularly polarized light," Proc. SPIE 11363, 1136304 (2020).

[24] Chue-Sang, J., Gonzalez, M., Pierre, A., Laughrey, M., Saytashev, I., Novikova, T., and Ramella-Roman, J. C., "Optical phantoms for biomedical polarimetry: a review," J. Biomed. Opt. 24(3), 1-12 (2019).

[25] Borovkova, M., Peyvasteh, M., Dubolazov, O., Ushenko, Y., Ushenko, V., Bykov, A., Deby, S., Rehbinder, J., Novikova, T., and Meglinski, I., "Complementary analysis of Mueller-matrix images of optically anisotropic highly scattering biological tissues," J. Eur. Opt. Soc.-Rapid Publ. 14, 20 (2018).

[26] Ossikovski, R., "Analysis of depolarizing Mueller matrices through a symmetric decomposition," J. Opt. Soc. Am. A 26(5), 1109-1118 (2009).

[27] Vizet, J. and Ossikovski, R., "Symmetric decomposition of experimental depolarizing Mueller matrices in the degenerate case," Appl. Opt. 57(5), 1159-1167 (2018).

[28] Ossikovski, R., Anastasiadou, M., Ben Hatit, S., Garcia-Caurel, E., and De Martino, A., "Depolarizing Mueller matrices: how to decompose them?," Phys. Stat. Sol. (a) 205(4), 720-727 (2008).

[29] Cloude, S., "Conditions for the Physical Realizability of Matrix Operators in Polarimetry," Proc. SPIE Polarization Considerations for Optical Systems 1166, 177-185 (1989).

[30] Goldstein, D. H., [Polarized Light - Third edition], CRC Press, Taylor and Francis (2010).

[31] Gil-Perez, G. J. and Ossikovski, R., [Polarized Light and the Mueller Matrix Approach], CRC Press, Taylor and Francis (2016).

[32] Lihong, V. W. Hsin, W., [Biomedical optics, principles and imaging], Wiley and Sons, New Jersey (2007).

[33] Cheong, W., Welch, A., and Prahl, S., "A review of the optical properties of biological tissues," IEEE J. Quantum Electron. 26, 2166-2185 (1990).

[34] Zherebtsov, E., Dremin, V., Popov, A., Doronin, A., Kurakina, D., Kirillin, M., Meglinski, I., and Bykov, A., "Hyperspectral imaging of human skin aided by artificial neural networks," Biomed. Opt. Express 10, 3545-3559 (2019).

[35] Bykov, A., Popov, A., Priezzhev, A., and Myllyla, R., "Multilayer tissue phantoms with embedded capillary system for oct and doct imaging," Proc. SPIE 8091, 80911R (2011).

[36] Wrobel, M., Popov, A., Bykov, A., Kinnunen, M., Jedrzejewska-Szczerska, M., and Tuchin, V., "Measurements of fundamental properties of homogeneous tissue phantoms," J. Biomed. Opt. 20(4), 045004 (2015).

[37] Wrobel, M., Popov, A., Bykov, A., Kinnunen, M., Jedrzejewska-Szczerska, M., and Tuchin, V., "Multilayered tissue head phantoms for noninvasive optical diagnostics," J. Innov. Opt. Heal. Sci. 8(3), 1541005 (2015).

[38] Moffitt, T. P., Chen, Y.-C., and Prahl, S. A., "Preparation and characterization of polyurethane optical phantoms," J. Biomed. Opt. 11(4), 041103 (2006).

[39] Van Eeckhout1, A., Garcia-Caurel, E., Ossikovski, R., Lizana, A., Rodríguez, C., González-Arnay, E., and Campos, J., "Depolarization metric spaces for biological tissues classification," J. Biophotoncis 13(8), e202000083 (2020).

[40] Van Eeckhout, A., Lizana, A., Garcia-Caurel, E., J., G., Ossikovski, R., and Campos, J., "Synthesis and characterization of depolarizing samples based on the indices of polarimetric purity," Opt. Lett. 42(20), 4155-4158 (2017). 